perimental class in physical culture was formed of valves. The prognosis in these cases depends on the twelve men, who for a period ranging from one to power of compensation, and is better in regurgitation two years, had made no appreciable progress in their than in stenosis. Mitral disease at middle life, or school work, and who seemed incapable of prolonged subsequent thereto, is generally secondary to some mental effort, yet could not, strictly speaking, be other form of cardiac disease, and the prognosis is considered mentally unsound, or representatives of a bad.

class known as feeble-minded; with the object of Disease of the aortic valves generally occurs in ascertaining, if possible, if physical culture, as com- adult life, probably owing to the tendency toward prised in frequent baths and massage, and daily calis- inflammation of the aorta, immediately around the thenics under the care of a competent instructor, sinuses of Valsalva, during this period.

would not result at least in the partial awakening and stimulation of dormant mental power. Increased mental activity rather than muscular development was to be the gauge of success or failure of the experiment.

Dr. H. D. Wey, ${ }^{4}$ physician to the Reformatory, in reporting the results of the experiment, says that to those who are thrown in daily contact with the men, a mental awakening was apparent. They became interested in their studies and strove to appear to the best advantage in the school-room. Their advancement in their studies was not steadily onward, but rather intermittently progressive. It will be interesting to note in the future, as Dr. Wey remarks, whether the good results are permanent; but whether they are or not, the step is one in the right direction, and is to be commended to penologists everywhere.

(To be concluded.)

PROGNOSIS IN VALVULAR LESIONS OF THE HEART.

Read before Chicago Pathological Society, December, 1887 . BY JOSEPH M. PATTON, M.D., OF CHICAGO.

The prognosis here is better in stenosis, than in regurgitation, aside from the liability to cerebral embolism, which Niemeyer limits to cases of regurgitation, I believe that embolism is not of very frequent occurrence as I have met with only one instance, and that in a case of stenosis in a girl I 2 or I 4 years of age.

Second, as to the physical changes in the heart effected by the lesion. And in this connection cardiac hypertrophy is not to be considered as a diseased condition, but as the necessary and only remedy which Nature can give to a pump whose power is being overtaxed by reason of a faulty valve.

In mitral regurgitation there is first dilatation of the left auricle, but this is generally slight, as the intra-auricular pressure is not very much augmented. The increased pressure in the pulmonary circulation is immediately relieved by hypertrophy of the right ventricle, which is a simple hypertrophy, and not eccentric, as is generally stated. When this compersation takes place the prognosis is good, and remains so until the hypertrophy becomes eccentric from degeneration of the right ventricle. The muscle being no longer able to withstand the increased intra-ventricular pressure, the prognosis then becomes grave.

The most interesting question in connection with cardiac valvular lesions, and one that requires the most accurate knowledge of the physical condition of the heart, and of the changes that have taken place, and of those which are yet to follow, is the question of prognosis.

The incurability of organic disease of the heart, confines the management of these cases to one object, namely, how long can the heart be made to perform its function? To this end it is necessary that an intelligent prognosis be made, based on the following points: First. The seat of the lesion and its character. Second, The physical changes already effected by the lesion. Third, The histological changes resulting from these altered physical relations.

The careful study of these questions, and of the liability to further changes, both physical and pathological, in which any or all of the above-named conditions may be factors, will enable us to make a reliable and scientific prognosis. The effect of location on the prognosis depends on the time of life at which the trouble begins, and on the character of the lesion.

In early life the mitral valve is generally the one at fault, and the lesion is most often regurgitant. This is due to the fact that mitral disease in young subjects is generally secondary to acute endocarditis, and to the high blood pressure on the under side of these

\footnotetext{
4 Annual Report of the New York State Penitentiary, at Elmira, for the year ending September 30,1886 .
} monary veins throws the blood into the left ventricle with more than usual force, hence there is slight dilatation and hypertrophy of this ventricle, which equalizes the arterial circulation. In some cases complicated by extensive myocarditis, dilatation of the left auricle, and ventricle, may be so rapid that the patient dies before compensatory hypertrophy can be obtained. In a recent case of this nature in a child 8 years old, the heart muscle was very weak from myocarditis. The patient was very anæmic, yet under treatment the improvement was very slow, but steady for several weeks, when, after taking a long walk and overtaxing the heart muscle, rapid dilatation took place and the child died in a week or ten days.

In mitral stenosis there is dilatation of the left auricle from the increased pressure necessary to force the blood through the constriction, and though the right heart compensates for the obstructed pulmonary circulation, the prognosis is rendered more grave than in regurgitation, because of the over-distension of the left auricle, which is but moderately supplied with muscular tissue, and is unable to withstand it.

In aortic disease the left ventricle furnishes the compensating power. In stenosis, its first effect is hypertrophy of the ventricle. This fully controls the interference with the blood current, and all goes well so long as the muscle retains its integrity. 
The prognosis at this stage is good, as a patient may remain in this condition for many years, totally ignorant of any cardiac trouble. Sooner or later muscular degeneration sets in, as evinced by-ventricular dilatation, as the muscle is now no longer able to withstand the blood pressure, and as the dilatation becomes advanced the mitral ring is stretched, insufficiency of that valve results, and this gives an obstructed pulmonary circulation at a time when muscular degeneration prevents compensation by the right heart, therefore, as soon as mitral regurgitation occurs in these cases, the prognosis gets grave.

In aortic regurgitation the first effect is dilatation from the direct force of the reflex current impinging on the walls of the left ventricle while it is relaxed, also, from overdistension by the addition of the regurgitated blood to that normally received from the left auricle. This is followed by hypertrophy, which is eccentric. The prognosis at this stage is not as favorable as in the same stage of stenosis, that is, when compensation is obtained, for the reason that in stenosis when the hypertrophy has fully overcome the obstruction at the valve it may remain stationary so long as there is no change at the valve, or in the integrity of the muscle, while in regurgitation the distending force is constantly present, and the hypertrophy must be progressive. It is for this reason that we find such immense left ventricles in some cases of aortic regurgitation. As soon as the hypertrophy fails to arrest the dilatation, mitral regurgitation ensues, as in stenosis, but is reached in much less time, as a rule, hence the prognosis is even more unfavorable than in the same stage of aortic stenosis.

The statement made by some authors that hypertrophy of the right heart in disease of the left side, and of the left ventricle in aortic stenosis, is eccentric, I believe to be erroneous. Niemeyer claims that simple hypertrophy is rare, and only obtained in such interference with the circulation as is given in Bright's disease or chronic asthma, or bronchitis. I do not recognize any difference between the effect on the left ventricle from the increased vascular pressure in Bright's disease, and the effect on the right ventricle in pulmonary obstruction from mitral regurgitation, or on the left ventricle in aortic stenosis before degenerative changes begin.

Histological Changes.-This consists in the hypertrophy of the muscular tissue, which results from the increased nutrition to the heart muscle, as demanded by its extra labor, similar to like changes in other muscular tissues under like circumstances. I believe this to be a simple increase in volume of the primitive muscular fasciculus, and not a numerical increase as is claimed by many. This increase in volume of the fasciculus may be caused by swelling of the sarcous elements of which the fibrillæ are composed, or possibly, increase of the number of fibrillæ within the sarcolemma, if there were actual increase in the number of fasciculi, with their investing sarcolemma, there would also be increase in the number of muscular bundles with their perimysium, and after degeneration of the muscular fibre this would leave an excess of the areolar tissue of which the perimysium is composed. This I have not been able to see.
The pathological changes taking place are those of degeneration from defective nutrition, and are followed by dilatation of the organ, by reason of lessening of the resisting power of its muscle.

The interference with the coronary circulation by reason of the physical changes in the heart by atheromatous degeneration of the aorta or coronary arteries by inability of the arteries to carry enough blood to -supply the increased volume of muscle, causes a fatty degeneration of the primitive muscular fasciculi. The endo-cardiac blood pressure now exceeds the contracting power of the muscle and rapid dilatation follows.

The prognosis then, in a heart that has been hypertrophied, becomes grave with the appearance of symptoms indicating dilatation, for now it is a question of only a short time until asystolism of the heart is developed, and under such conditions the heart cannot long perform its function.

A word as to the danger of sudden death from organic disease of the heart. I do not believe this danger is sufficiently imminent to warrant us in placing it among the elements of an unfavorable prognosis. There is no influence in the heart, per se, tending to cause sudden cessation of its action. Cerebral embolism, it is true, may cause sudden death, but this, as already shown, is not frequent.

The formation of a ventricular clot from healthy blood is not possible, except when the heart has developed asystolism, in which case the prognosis is already rendered grave, because of the physical condition of the heart. I have never seen a sudden death from organic heart disease where the diagnosis was assured and the cause of death referable to the heart. And were the actual cause known of the many sudden deaths which are laid at the door of this hardworking and long-suffering organ, I believe the vast majority would be found due to causes extraneous to the heart, probably some circulatory disturbance, or tissue degeneration of the nervous centers, causing a lack of the proper innervation of the heart. The fact at least remains, that in organic disease of the heart sudden and unexpected death is a marked exception to the general termination, and if this be true in diseased conditions, what cogent reason can be given for attributing sudden death to the heart, in persons who have never given any evidence of cardiac disease.

From the foregoing we would derive the following conclusions :

The prognosis when compensation is obtained is better in aortic stenosis and in mitral regurgitation than in aortic regurgitation, or mitral stenosis. A person with either of the former lesions, moderately severe and fully compensated for, may live 20 or 30 years and suffer no inconvenience therefrom.

In aortic stenosis intercurrent disease of the lungs is not so dangerous as in mitral regurgitation, hence the prognosis in the former is somewhat better.

In aortic regurgitation, and in mitral stenosis, the prognosis is not so good, because the eccentric hypertrophy in the former, and the dilatation of the left auricle in the latter, renders perfect compensation difficult. 
The more pronunced the lesion the more grave the prognosis, in direct proportion to the increase in intra-cardiac pressure.

Double lesions at one valve, or two distinct lesions at different valves, which have begun at the same time, render the prognosis grave in direct proportion to the increased liability to dilatation of the different cavities.

\section{SALICYLATE OF AMMONIUM FOR FEVERS.}

BY D. M. WICK, M. D.,

OF NEW HARTFORD, IOWA.

Last winter, in February, I was treating L. P., a boy of 6 years, for remittent fever, the temperature varying from ${ }_{10} 2^{\circ}$ to $\mathrm{rO}_{3}^{\circ}$. I, began with the classical course : hydrarg. chlor. mitis to arouse the secretions, quinine as an antiperiodic, and veratrum and spts. æeth. nitrosi as a febrifuge. At the close of the secon 1 day of treatment, he broke out on face and neck with a bright scarlatinoid eruption, resembling very much that of scarlet fever. The parents became greatly alarmed, fearing that the boy had scarlet fever. Assuring them that he had not, and that the eruption was caused by the powders, I continued the same treatment. The next day the eruption was still more diffused over the whole surface of body, with intolerable and incessant itching and fever as high as at first visit. For another day (the fourth) the same treatment was persisted in, only to aggravate the erythema, cause cdema of face, hands and feet, and with the itching, that had persisted from the first appearance of the quinine exanthem, made my little patient more restless and miserable. The fever had now increased to $103^{\circ}$ morning and $104^{\circ}$ evening. I had never used salicylate of ammonium, but thought this would be an opportunity to test its value as an antipyretic. I had read the papers of Drs. Jackson, Barnett and Sullivan. Leaving off all the former medicines, I dissolved 3 ss salicylate ammonium in $\xi_{i j}$ aquæ and ordered I teaspoonful every two hours.

I saw the patient again in eight hours, and found him with temperature reduced to $99 \cdot 5^{\circ}$, resting quietly and sweating profusely. Continued the dose the same and lengthened the interval to four hours. Next day the temperature was normal, tongue becoming clear, eruption disappearing, and the boy feeling so well that he wanted to be up and dressed. He made a rapid recovery.

The next case in which $I$ used the new drug was also one of remittent fever. A boy, 5 years old, had been sick nearly one week when first seen. I put him on the orthodox treatment, and continued it one week, during which time his fever was never below $102^{\circ}$ nor above $104^{\circ}$. He had a hot, dry skin all the time.

I again dropped all other drugs and gave salicylate of ammonium, gr. ij, in water every two hours. In about eight hours his temperature fell to $99^{\circ}$ and he was bathed in perspiration. Continued the dose once in four hours. Saw the case next day and found no fever, tongue clean, skin moist, and the boy in every respect improved. He had no relapse, and was soon able to be around.
May P., a girl of I I years, had measles. The dis. ease pursued its usual course and the eruption receded as in other cases. But the fever, which had ranged high throughout (from $12^{\circ}$ to $104^{\circ}$ ) continued. An active diarrhoea set in, her tongue was dry and heavily coated, and sordes was on the teeth. She was now in a semi-delirious condition, with a temperature of $105^{\circ}$. I had no trouble in controlling the bowels with mineral acids and small doses of opiates. Despite the frequent bathing, quinine, gelsemium and aconite, the fever kept on as high as ever.

After five days of this course she was no better, save that her bowels were more quiet. The skin was dry, temperature $105^{\circ}$, with considerable nervous disturbance, and still semi-conscious. As the case was assuming a very serious aspect, I again resorted to the ammonia treatment. Dropping all other medicines as in the former cases, I ordered 2 grains every hour until she should sweat. The nurse told me the next morning that after giving the fourth dose she began to sweat, and by the time the fifth was to be given, her clothing was so thoroughly saturated that the time was lengthened to four hours. Her temperature was normal, pulse soft and regular, though weak, tongue moist for the first time in five days and showing evidence of becoming clean. From this time she gained rapidly without one unfavorable symptom, and in one week was up, and soon recovered.

Netta B., a frail and delicate girl of 7 years, was attacked with whooping-cough, which ran a severe course for two weeks and then, insidiously, the case took on broncho-pneumonia. Temperature rose to $104^{\circ}$, breathing hurried, oppression excessive, the lips and face turgid and cyanotic, pulse rapid. Gave her a mixture of carbonate of ammon., acetat. potass., syr. ipecac, and tinct. nux vomica as a cardiac and respiratory stimulant; alternating with small doses of quinine. After using this treatment four days, the fever not abating and other symptoms becoming worse, I again quit all other drugs and admininistered 2 grains of salicylate of ammonium every two hours until free perspiration occurred, which was after the sixth dose. The fever subsided, respiration became easy and expectoration abundant. The temperature did not return above $\mathrm{roI}^{\circ}$ for several days. Although it is a peculiarity of broncho-pneumonia to lull, and give a deceptive promise of amendment, I am convinced that the remedy acted promptly in lessening the fever; for after the second and third invasion of the bronchial tree, with temperature of $104^{\circ}$ and $105^{\circ}$ (that after four weeks proved fatal), the salicylate of ammonium each time brought the temperature down to $101^{\circ}$ or $101.5^{\circ}$

I have given this form of ammonium in other cases, but did not have the opportunity to watch its effects as in the above. In the first case reported, I was forced to seek some other remedy, from the unpleasant effects of quinine. In the second, I had not yet become interested enough in the new drug to give up the old form of medication; while in the third, I thought the little girl too dangerously sick to try new remedies. In the fourth I was desirous to test its virtues only after first using the ordinary plan of treatment. 